\title{
Raman-Kerr comb generation based on parametric wave mixing in strongly driven Raman molecular gas medium
}

\author{
Aurélien Benoît, ${ }^{1}$ Anton Husakou, ${ }^{2}$ Benoît Beaudou, ${ }^{3}$ Benoît Debord, ${ }^{1,3}$ Frédéric Gérôme, ${ }^{1,3}$ and Fetah Benabid ${ }^{1,3, *}$ \\ ${ }^{1}$ GPPMM Group, Xlim Research Institute, CNRS UMR 7252, University of Limoges, Limoges, France \\ ${ }^{2}$ College of Max Born Institute, Forschungsverbund Berlin e.V., Rudower Chaussee 17, 12489 Berlin, Germany \\ ${ }^{3}$ GLOphotonics SAS, 123 Avenue Albert Thomas, 87060 Limoges Cedex, France
}

(Received 20 March 2019; accepted 4 March 2020; published 9 April 2020)

\begin{abstract}
We report on experimental and theoretical demonstrations of an optical comb spectrum based on a combination of cascaded stimulated Raman scattering and four-wave mixing mediated by Raman-induced nonresonant Kerrtype nonlinearity. This combination enabled us to transform a conventional quasiperiodic Raman comb into a comb with a single and smaller frequency spacing. This phenomenon is achieved using a hollow-core photonic crystal fiber filled with 40 bars of deuterium and pumped with a high-power picosecond laser. The resultant comb shows more than 100 spectral lines spanning over $220 \mathrm{THz}$ from $800 \mathrm{~nm}$ to $1710 \mathrm{~nm}$, with a total output power of 7.1 W. In contrast to a pure Raman comb, a $120 \mathrm{THz}$ wide portion of the spectrum exhibits denser and equally spaced spectral lines with a frequency spacing of around $1.75 \mathrm{THz}$, which is much smaller than the lowest frequency of the three excited deuterium Raman resonances. A numerical solution of the generalized nonlinear Schrödinger equation in the slowly varying envelope approximation provides very good agreement with the experimental data. The additional sidebands are explained by cascaded four-wave mixing between preexisting spectral lines, mediated by the large Raman-induced optical nonlinearity. The use of such a technique for coherent comb generation is discussed. The results show a route to the generation of optical frequency combs that combine large bandwidth and high power controllable frequency spacing.
\end{abstract}

DOI: 10.1103/PhysRevResearch.2.023025

\section{INTRODUCTION}

The generation of optical combs is turning into an extremely important topic in several scientific and technological fields, such as waveform synthesis [1], optical clocks [2], communication systems [3], and biomolecular imaging [4]. Historically, generation of optical combs was associated with mode-locked lasers [5] or with high harmonic generation using high-peak-power and ultrashort-pulse laser sources $[1,6]$. The former has matured to be the cornerstone in optical frequency metrology thanks to the outstanding frequency stability of the comb spectral components [7], and the latter is a powerhouse in synthesizing attosecond pulses in vacuum ultraviolet (VUV) and soft x-ray experiments thanks to its multioctave bandwidth [8]. During the last two decades, photonics solutions to generate optical combs have emerged with potential to address current limitations such as compactness or multioctave comb generation in more convenient spectral ranges. Among these, we list Kerr optical nonlinearity based combs, which are generated by microresonators and pumped with a continuous-wave laser [9]. Here, the generated

\footnotetext{
*Corresponding author: f.benabid@xlim.fr

Published by the American Physical Society under the terms of the Creative Commons Attribution 4.0 International license. Further distribution of this work must maintain attribution to the author(s) and the published article's title, journal citation, and DOI.
}

spectrum can be up to one octave wide and the frequency spacing of its components, set by the microresonator diameter, ranges from a few hundred GHz up to $1 \mathrm{THz}$. Another means of generating a discrete spectrum is based on stimulated Raman scattering (SRS) in gas-filled hollow-core photonics crystal fiber (HC-PCF) [10]. Compared to mode-locked laser or microresonator mechanisms, the Raman-gas-filled HC-PCF based combs stand out with their large bandwidth [10], which can span more than five octaves from the ultraviolet to midinfrared range, and with their high optical power and energy handling [11]. The generated spectra using Raman-gas-filled HC-PCF with nanosecond or picosecond pump pulses show intrapulse phase coherence [12] and are typically composed of lines with several frequency spacings exclusively set by the rotational and/or vibrational Raman resonances $[10,11,13,14]$. Consequently, the typical spectral structure of a HC-PCF based Raman comb consists of a central frequency $v_{0}$ set by the pump laser, and a cascade of sidebands with frequencies in the form of $v_{l, m, n}=v_{0}+l v_{R 1}+m v_{R 2}+n v_{R 3}$ for the case of a medium with three Raman transitions. Here, $l, m, n$ are integers corresponding to the negative (Stokes) and positive (antiStokes) orders of the Raman transitions with frequencies $v_{R 1}$, $v_{R 2}$, and $v_{R 3}$ respectively. Note that the spectral structure of such SRS comb is set by resonant third-order susceptibility in the vicinity of the Raman resonance [15], with no measurable influence from the nonresonant nonlinear susceptibility (i.e., Kerr effect) [16]. As such, the HC-PCF based Raman comb suffers from two major drawbacks that hinder their use in a number of applications. It exhibits multiple frequency spacing 
(quasiperiodic comb), and its Raman frequency is too large ( $>10 \mathrm{THz}$ for most of the representative molecular gases).

In this paper, we show an approach to overcome this limit of Raman combs and to generate a small and single frequency spaced comb in Raman gas media of the form $v_{q}=v_{0}+q \Delta$. The approach relies on using a Raman gas containing at least two nearly commensurable resonance frequencies, so that, for example, $v_{R 2}$ and $v_{R 3}$ can be written as $v_{R 2}=N \Delta+\delta$ and $v_{R 3}=M \Delta+\delta^{\prime}$ with $N$ and $M$ being positive integers, $\delta, \delta^{\prime} \ll \Delta$. The proof of principle of the approach is undertaken by experimentally and theoretically demonstrating the generation of a wide comb in a Raman gas-filled HC-PCF that exhibits spectral lines with a frequency spacing as low as 1.75 THz. The observed spectrum, which is generated from a high-pressure deuterium molecular gas, consists of up to 100 spectral lines covering a width of $220 \mathrm{THz}$. In addition to lines associated with the three excited Raman resonances of deuterium at frequencies of $89.6,12.4$, and $5.3 \mathrm{THz}$, a good part of spectrum includes a denser comb of 52 lines covering a range of $120 \mathrm{THz}$ with a frequency spacing of 1.75 THz. We attribute these denser spectral lines to an action of Raman-induced parametric four wave-mixing (FWM) (i.e., the out-of-resonance contribution of the Raman susceptibility) and to a FWM process between close Raman sidebands to generate frequency components outside the Raman resonance frequencies.

\section{EXPERIMENTAL SETUP AND RESULTS}

The experimental setup consists of a high-power picosecond pulsed $\mathrm{Yb}$-fiber laser coupled into a photonic microcell (PMC) comprising a length of HC-PCF whose ends are attached to gas cells [17]. The laser emits at $1030 \mathrm{~nm}$, has an average power of $16 \mathrm{~W}$, a repetition rate of $1 \mathrm{MHz}$, pulse duration of $\tau_{\mathrm{p}}=27 \mathrm{ps}$, and a self-phase modulation broadened spectrum with a bandwidth of $200 \mathrm{GHz}$ [18]. The PMC is filled with deuterium molecules $\left(\mathrm{D}_{2}\right)$ at a pressure of 40 bars. The choice of $\mathrm{D}_{2}$ is motivated by the frequency values of its two ro-vibrational Raman transitions. At room temperature, $\mathrm{D}_{2}$ gas is a $2: 1$ molecular mixture of orthodeuterium $\left(\mathrm{o}-\mathrm{D}_{2}\right)$ and para-deuterium $\left(\mathrm{p}-\mathrm{D}_{2}\right)$. It exhibits three Raman resonances: a vibrational one around $v_{R 1}=89.6 \mathrm{THz}$ with a steady-state Raman gain of $0.37 \mathrm{~cm} / \mathrm{GW}$, and two ro-vibrational ones at $v_{R 2}=12.44 \mathrm{THz}$ from o- $\mathrm{D}_{2}$ [19] and at $v_{R 3}=5.33 \mathrm{THz}$ from $\mathrm{p}-\mathrm{D}_{2}$ [20] with gains of $\sim 0.12$ and $\sim 0.05 \mathrm{~cm} / \mathrm{GW}$ respectively. As a result, the deuterium Raman shifts for para- and ortho- molecules are close to being commensurable, with their ratio being equal to 3:7 within less than $0.1 \%$ of relative error. As such, we have $5.33 \mathrm{THz} \sim 3 \Delta$, and $12.44 \mathrm{THz} \sim 7 \Delta$, where $\Delta=\sim 1.78 \mathrm{THz}$ coincides with the $\left|v_{R 2}-2 v_{R 3}\right|$ value, which is also the frequency spacing between the Raman lines $v_{l, m+1,-1}$ and $v_{l, m, 1}$, and is three times smaller than the smallest Raman frequency. The impact of this peculiarity will be apparent later. Furthermore, the gains of the $\mathrm{o}-\mathrm{D}_{2}$ and $\mathrm{p}-\mathrm{D}_{2}$ ro-vibrational resonances have closer values (2:1 ortho to para ratio of gains) than does hydrogen gas (3:1 ratio) [21], thus favoring the excitation of $\mathrm{p}-\mathrm{D}_{2}$ Raman resonance when compared to $\mathrm{H}_{2}$. Finally, operating at a pressure as high as 40 bars ensures higher Raman gain but broader gain linewidth, typically $\sim 10 \mathrm{GHz}$.
The latter is notably useful for fulfilling the above-mentioned commensurability. The PMC fiber consists of a $3 \mathrm{~m}$ long inhibited-coupling single-ring tubular-lattice HC-PCF [22] with an inner diameter of $40 \mu \mathrm{m}$ [Fig. 1(a)].

The fiber transmission loss in the visible-infrared range is characterized by an ultrawide (over one octave) band from 600 to $1200 \mathrm{~nm}$ with loss around $11 \mathrm{~dB} / \mathrm{km}$ [Fig. 1(b)]. Figure 1(b) presents also the evolution of the refractive index as a function of the wavelength. Figures 1(c) and 1(d) show the used fiber spectra of the group index and the dispersion respectively. A detailed account of all the optical properties of the HC-PCF is given in [22]. The laser beam is coupled into the PMC in a manner to match its beam size with the fiber fundamental core mode. Inspection of the fiber output beam profile over the operating spectral range of 800 $1600 \mathrm{~nm}$ shows single mode propagation [inset of Fig. 1(e)]. Figure 1(e) presents the typical PMC output spectrum when excited with $16 \mathrm{~W}$ average power pump laser. The power of the generated and transmitted spectrum was measured to be 7.1 W. The spectrum spans a wide range from 170 to $390 \mathrm{THz}$ and exhibits a large number of lines in a comblike structure with more than 100 lines. The spectral components reveal that two purple-colored lines correspond in their frequency shifts $(89.63 \mathrm{THz})$ to the vibrational Stokes $v_{-1,0,0}$ and antiStokes $v_{1,0,0}$ lines of the pump. The vibrational comb lines are accompanied by the comb of blue lines, which appear around both pump and the first-order vibrational Stokes and anti-Stokes lines. The blue lines are readily associated with ortho-ro-vibrational Raman resonances, as corroborated by their frequency offset from their associated pump (average experimental shift of $12.45 \pm 0.06 \mathrm{THz}$ as compared to the theoretical value of $12.44 \mathrm{THz}$ ). Similarly, the green lines correspond to the Stokes and anti-Stokes sidebands of either the purple or the blue lines, and are generated by the pararo-vibrational Raman resonance (average experimental shift of $5.30 \pm 0.20 \mathrm{THz}$ as compared to the tabulated value of $5.33 \mathrm{THz}$ ). Finally, the spectrum includes red lines, which, even though they can be described in terms of the Raman frequency expression $v_{l, m, n}$, are generated by a more subtle mechanism than the simple cascaded Raman picture effect, as discussed below. Limiting the further discussion to only the pump laser sidebands of orders $(0, m, n)$, we see that this part of the comb extends over 220-340 THz, and its Raman frequencies take the form of $v_{0, m, \pm 1}$ with no overlapping lines from the vibrational Stokes and anti-Stokes sidebands. Remarkably, the spectrum stands out with equally spaced spectral components, with a frequency spacing $\Delta=1.75 \pm$ $0.05 \mathrm{THz}$, which is also equal to $v_{R 2}-2 v_{R 3}$, the spacing of two Raman lines located at $v_{0, m+1,-1}$ and $v_{0, m, 1}$. Thus, this part of the spectrum is transformed from a quasiperiodic Raman comb into a single-frequency comb whose component takes the form $v_{q}^{-}=v_{0}+q \Delta$, with $q$ being an integer. Figure 1(f) shows a close-up of a portion of this spectrum, which is also representative of any range between $v_{0, m, 0}$ and $v_{0, m+1,0}$. It shows that, in addition to Raman blue lines $\left(v_{0,-2,0}\right.$ and $\left.v_{0,-1,0}\right)$ and green lines $\left(v_{0,-2,1}\right.$ and $\left.v_{0,-1,-1}\right)$, we have four red lines (labeled $v_{F W M 1}, v_{F W M 2}, v_{F W M 3}, v_{F W M 4}$ ). Also, Fig. 1(f) shows weak orange lines that are believed to originate as a cascade of the same phenomenon behind the generation of the red spectral lines. 

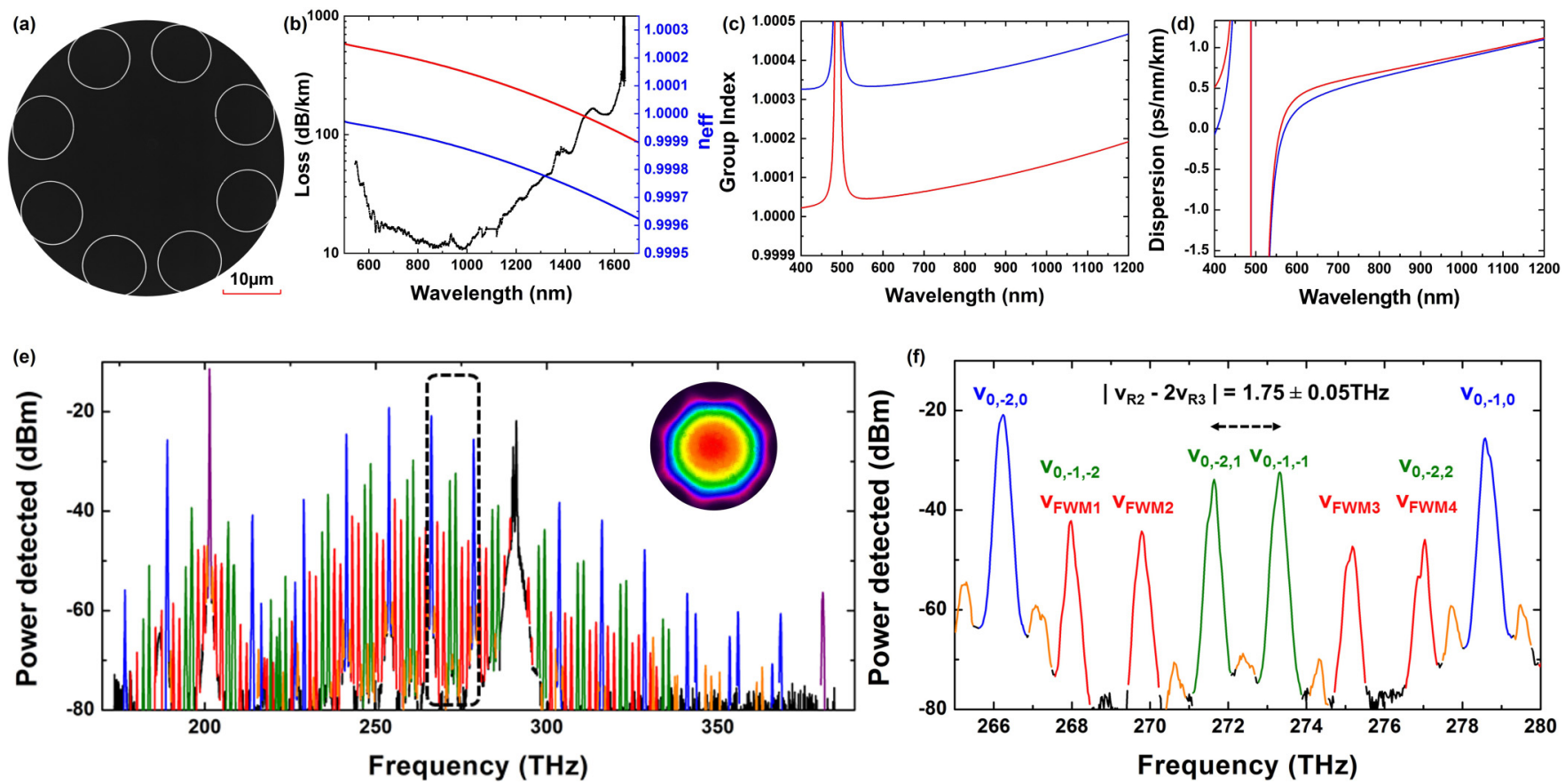

FIG. 1. (a) Scanning electron microscope image of the inhibited-coupling single-ring tubular-lattice HC-PCF used for this experiment. (b) Evolution of losses (black curve) and effective refractive index in the vacuum (blue curve) and in hydrogen (red curve) as a function of wavelength. (c) Evolution of group index as a function of the wavelength in the vacuum (blue curve) and in hydrogen (red curve). (d) Evolution of dispersion as a function of wavelength in the vacuum (blue curve) and in hydrogen (red curve). (e) Output spectrum after a $16 \mathrm{~W}$ power pump propagation in a $3 \mathrm{~m}$ long HC-PCF filled with 40 bars of deuterium. The pump laser line is identified by black color, the vibrational Stokes and anti-Stokes lines by purple, the Stokes and anti-Stokes lines due to ortho-ro-vibrational Raman resonance by blue, the Stokes and anti-Stokes lines due to para-ro-vibrational Raman resonance by green, and four-wave-mixing generated sidebands by red. Inset: Near-field intensity distribution of the output beam. (f) Zoomed-in spectrum of the black-dashed rectangle.

We start by recalling that the generation of the comb components is chronologically ordered by the Raman gain strength. Consequently, the vibrational lines (purple lines) appear first, then the ortho-ro-vibrational (blue lines) [denoted by Stage (i) in Fig. 2], and finally the para-ro-vibrational Raman lines $v_{0, m, \pm 1}$ (green) [Stage (ii) in Fig. 2]. The latter are sidebands of the black line $v_{0,0,0}$ (pump), and blue lines $v_{0, m, 0}$ (ortho-ro-vibrational Raman). This sequence was corroborated theoretically (see below). In order to determine the dominating mechanism between cascaded SRS and the Kerr effect in the red lines' generation, we qualitatively assessed the Raman gain in the transient regime $[23,24]$ and Kerr gain [25]; or alternatively their associated characteristic lengths (see Appendix A: Competition between SRS and FWM). The results show that the Kerr characteristic length is 110 shorter than the Raman characteristic length, thus indicating that the generation of lines at $v_{F W M 1}, v_{F W M 2}, v_{F W M 3}, v_{F W M 4}$ is dominated by Kerr nonlinearity. Consequently, the red lines result from a cascaded parametric FWM process, which is further facilitated by the combination of three favorable circumstances: (i) the near-commensurability of $v_{R 2}$ and $v_{R 3}$, (ii) the small frequency spacing between two adjacent green lines, and (iii) the PMC weak dispersion (see below and Fig. 3). Below, we discuss the involved comb lines behind this wave mixing, and the origin of such a Kerr-type nonlinearity. These red-colored lines can result from wave mixing involving several combinations of $v_{0, m, n}$. For example, looking at the $v_{F W M 2}$ spectral line $269.8 \mathrm{THz}$ in Fig. 1(f), we identify several possible FWM processes to generate this line, like $v_{F W M 2}=$ $2 v_{0,-2,1}-v_{0,-1,-1}$ or $v_{F W M 2}=v_{F W M 1}+v_{0,-1,-1}-v_{0,-2,1}$ or $v_{F W M 2}=v_{0,-2,1}+v_{0,-2,0}-v_{F W M 1}$, and so on. Our aim now is to determine which of the possible FWM processes and spectral combinations lead to the generation of the red lines. Due to complicated dynamics involving high-order nonlinearities, the cascaded nature of the considered process, and the influence of phase matching, we found it useful to start by recalling the expression for the intensity of the new frequency $v_{1}$ by FWM process from frequencies $v_{2}, v_{3}$, and $v_{4}$ [26]:

$$
I_{1}=9 \pi^{2} \chi^{(3)^{2}}\left(v_{1}, v_{2}, v_{3}, v_{4}\right) I_{2} I_{3} I_{4} v_{1}^{2} \varepsilon_{0}^{2} \sin ^{2}(\Delta k z),
$$

where $\chi^{(3)}\left(v_{1}, v_{2}, v_{3}, v_{4}\right)$ is the frequency-dependent nonlinear susceptibility, $I_{1}$ to $I_{4}$ are the intensities at the participating frequencies, $z$ is the propagation length, and $\Delta k$ is the associated wave-number mismatch. Expression (1) shows three possible dominating effects in the generation of red spectral lines: (i) the strength of frequency-dependent nonlinear susceptibility (see Appendix B: Susceptibility in Raman-active medium), (ii) the powers of the participating lines, and (iii) the mismatch $\Delta k$ between the participating frequencies. Before trying to identify the leading effects in the observed FWM, we can easily disregard the nonresonant Kerr susceptibility, as the estimated characteristic length $L_{N L}=c /\left(2 \pi \cdot v_{0} \cdot I . n_{2}\right)$ necessary for the generation of FWM sidebands by the nonresonant Kerr process is $\sim 150 \mathrm{~m}$ for $n_{2}=0.3 \times 10^{-8} \mathrm{~cm}^{2} / \mathrm{TW}$, which is much larger than the fiber length. On the other 


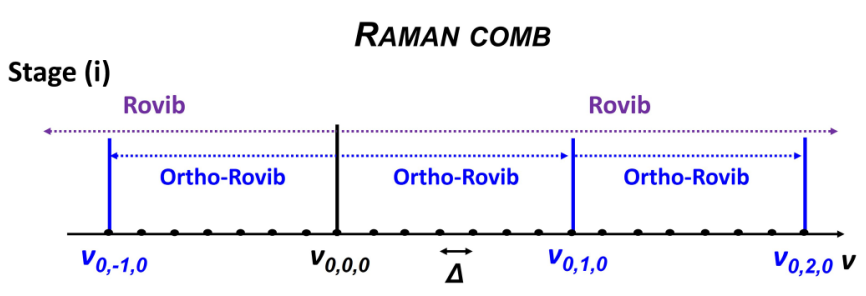

Stage (ii)
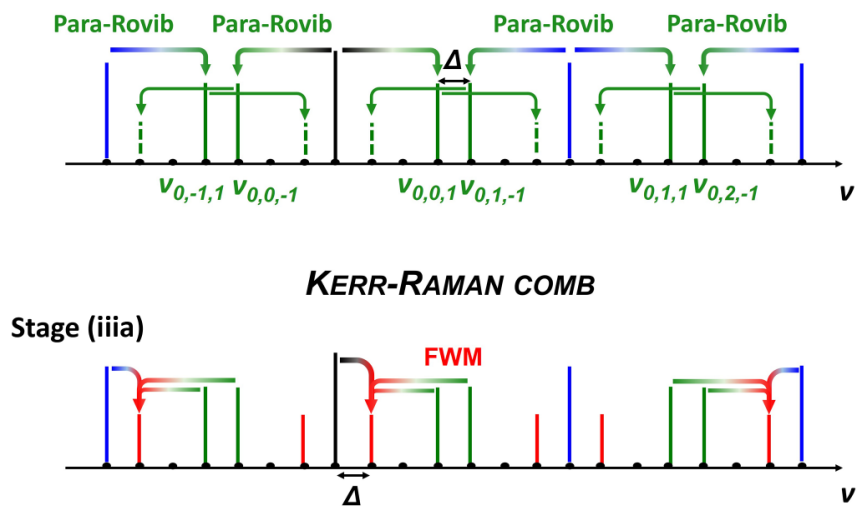

Stage (iiib)

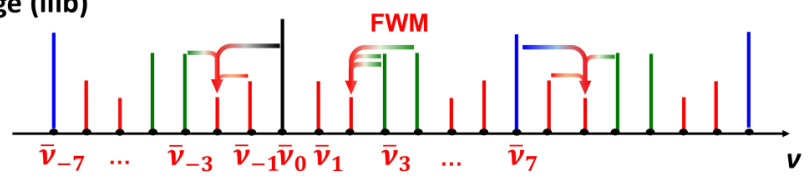

FIG. 2. Schematic sequence of the processes during the comb generation. The pump laser line is identified by black color, the vibrational Stokes and anti-Stokes lines by purple (not visible), the Stokes and anti-Stokes lines due to ortho-ro-vibrational Raman resonance by blue, the Stokes and anti-Stokes lines due to pararo-vibrational Raman resonance by green, and four-wave-mixing generated sidebands by red.

hand, we can calculate a rough estimate of the Raman-induced effective value of $n_{2, \text { Raman }}$ by comparing the characteristic length observed experimentally for the red lines with that of the Kerr effect. We obtain an effective value of $n_{2, \text { Raman }}=3 \times$ $10^{-8} \mathrm{~cm}^{2} / \mathrm{TW}$, which corresponds to the value of $5.5 \times 10^{-8}$ measured for $\mathrm{D}_{2}$ [27]. In Appendix $\mathrm{B}$, we derive a rigorous

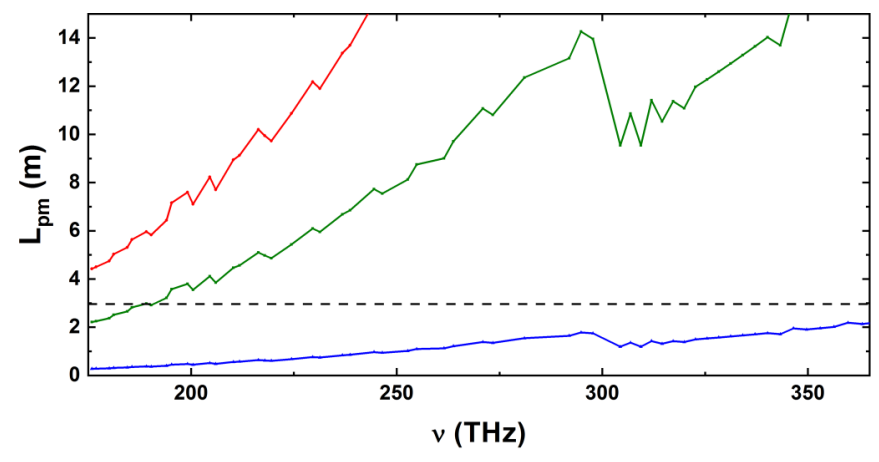

FIG. 3. The phase-matching lengths for the $v_{F W M 2}=2 v_{0,-2,1}-$ $v_{0,-1,-1}$ type of FWM process (red curve), for the $v_{F W M 1}=v_{0,-2,0}+$ $v_{0,-1,-1}-v_{0,-2,+1}$ type of FWM process (green curve), and for the $v_{F W M 1}=v_{0,-2,0}+v_{0,0,-1}-v_{0,-1,1}$ type of FWM process (blue curve). The dashed black line indicates the $3 \mathrm{~m}$ fiber length. expression for the nonlinear polarization in a Raman medium using the standard adiabatic elimination of the upper Raman states. The resultant expression is cumbersome to investigate; however, for the aim of current analysis it suffices to extract the terms that are not in resonance with the Raman transition, and keep the dominance from the remaining terms. The expression of a representative term is

$$
\begin{aligned}
P_{R-F W M}(t) & \\
= & \frac{N \mu_{j a}^{2}}{(8 \pi \hbar)^{3}}\left|\mu_{j a}\right|^{2} \\
& \times \sum_{q, q^{\prime} \neq q} \sum_{q^{\prime \prime}} \frac{E_{q}^{*} E_{q^{\prime}} E_{q^{\prime \prime}} e^{i 2 \pi\left(v_{q}-v_{q^{\prime}}-v_{q^{\prime \prime}}\right) t}}{\left(v_{q}-v_{q^{\prime}}\right)\left(v_{j}-v_{q^{\prime}}\right)\left(v_{j}-v_{q^{\prime \prime}}\right)} .
\end{aligned}
$$

This Raman induced nonresonant polarization clearly shows that the polarization responsible for the FWM process increases for fields with close frequencies due to the $v_{q}-v_{q^{\prime}}$ term appearing in the denominator (see Appendix B for notations). Based on the above, we conclude that the FWM process contributing to the red line generation stems from Raman induced enhanced nonlinearity. Second, by observing that the ratio of the values of the susceptibility $\chi^{(3)}\left(v_{1}, v_{2}, v_{3}, v_{4}\right)$ for two different possible FWM processes is determined by the ratio of the smallest difference between the participating frequencies, we can deduce that, among all the possible spectral lines fulfilling the required energy conservation, only those with small frequency difference dominate. For example, we found this ratio to have a maximum value of 2 for the relevant FWM processes from neighboring lines, while mechanisms involving non-neighboring lines will be suppressed by the factor in the denominator. Further shortlisting of the dominant FWM frequency combinations can be done using the phasematching condition.

Figure 3 shows the phase-matching length, $L_{p m} \equiv \pi . \Delta k^{-1}$, for three representative FWM frequency combinations. The broken line structures of the curves are due to the limited resolution of the fiber dispersion data. Also, the change in slope at $300 \mathrm{THz}$ is related to the dispersion spectral structure [see Fig. 1(d)]. The first two correspond to $v_{F W M 2}=$ $2 v_{0,-2,1}-v_{0,-1,-1}$ and $v_{F W M 1}=v_{0,-2,0}+v_{0,-1,-1}-v_{0,-2,1}$, respectively, and include frequencies from only neighboring lines. The third is of type $v_{F W M 1}=v_{0,-1,-1}+v_{0,0,1}-v_{0,-1,0}$, $v_{F W M 1}=v_{0,-2,0}+v_{0,0,-1}-v_{0,-1,1}$ containing larger spaced frequencies.

The results show that, for the majority of relevant processes from neighboring lines, $L_{p m}$ is longer than the fiber length, so that phase matching is fulfilled, and does not play a significant role in selecting the FWM mechanism except for suppressing contributions from non-neighboring lines. On the other hand, the difference between the intensities of the different line series is typically one order of magnitude. Therefore, we can conclude that the power of the participating frequencies will play the dominant role in selecting the FWM process, which provides the maximum contribution to the generation of new lines. Based on that, we expect that the strongest process which contributes to the generation of the $v_{F W M 1}$ line is $v_{F W M 1}=v_{0,-2,0}+v_{0,-1,-1}-v_{0,-2,1}$ process, while the subsequent $\nu_{F W M 2}=v_{0,-2,1}+v_{0,-2,0}-v_{F W M 1}$ and $\nu_{F W M 2}=$ $2 v_{0,-2,1}-v_{0,-1,-1}$ processes are mainly responsible for the 
generation of $v_{F W M 2}$ lines. These processes are indicated as Stage (iiia) and Stage (iiib) in Fig. 2. Other processes certainly provide additional and coherent contribution to the abovementioned ones, since the offsets of all frequencies are roughly multiples of $\Delta$. For deuterium gas we get a clear comblike spectrum with a step of approximately $\Delta=1.75 \mathrm{THz}$. The generation lines at $v_{F W M 3}$ and $v_{F W M 4}$ follows a process similar to that for $v_{F W M 1}$ and $v_{F W M 2}$. Finally, the spectrum shows very weak orange lines in Figs. 1(e) and 1(f), which are equally spaced from their adjacent lines by $\sim \Delta / 2$. Because of the same arguments as above, these lines result from further mixing of the lines. Due to their weakness, we do not find it worthwhile to identify their originating lines.

\section{NUMERICAL AND THEORETICAL APPROACHES}

To corroborate the above conclusions and to achieve a deeper understanding of the processes which govern the generation of FWM sidebands in Raman-gas medium, we have developed a numerical model. The simulation of the Raman comb generation was performed by numerically solving the generalized nonlinear Schrödinger equation in the slowly varying envelope approximation, which is written as [22,28]

$$
\begin{aligned}
\frac{\partial A(\omega)}{\partial z}= & i\left[k(\omega)-k\left(\omega_{0}\right)-\left.\left(\omega-\omega_{0}\right) \frac{\partial k(\omega)}{\partial \omega}\right|_{\omega_{0}}\right] A(\omega) \\
& +\frac{i \omega}{2 c \varepsilon_{0}} \sum_{j=2,3} P_{R, j}(\omega)
\end{aligned}
$$

where $A(\omega)$ is the field envelope in the spectral presentation, $k(\omega)$ is the wave number which includes gas dispersion (calculated using the Sellmeyer-type expressions), the waveguide dispersion (determined from [22]), the phase mismatch, and the fiber loss spectrum. The quantity $\omega_{0}$ is the pump central angular frequency and $P_{R, j}$ is the Raman polarization induced by the Raman transition $R, j$. In our case, we consider only the two ro-vibrational resonances of ortho- and para-deuterium, and ignore the vibrational transition. This choice is motivated by the large value of the vibrational Raman frequency, the higher fiber loss at $\nu_{ \pm 1,0,0}$, and correspondingly the lower number of sidebands generated by the vibrational Stokes and anti-Stokes fields. In this simulation, we have deliberately neglected the nonresonant Kerr response since it is weak in comparison to the nonresonant Raman response and to determine the role of Raman response in the nonresonant polarization. The Raman polarization is given by

$$
P_{R, j}(t)=A(t) \varepsilon_{0} \tilde{p}_{j}\left(\alpha_{\rho, j} \rho_{12, j}+\alpha_{w, j} w_{j}\right),
$$

where $\tilde{p}_{j}$ are pressures, $\rho_{12, j}$ and $w_{j}$ are the off-diagonal elements of the density matrix and the inversion, correspondingly, and $\alpha_{p, j}$ and $\alpha_{w, j}$ are coefficients which are related to the dipole moment of the deuterium molecules. The temporal evolution of the quantities $\rho_{12, j}$ and $w_{j}$ is described by standard two-photon two-level Bloch equations:

$$
\begin{gathered}
\frac{\partial \rho_{12, j}}{\partial t}=\rho_{12, j}\left[-\frac{1}{\tau_{j}}+i \omega_{R j}+\tilde{\alpha}_{1, j}|A(t)|^{2}\right]+\alpha_{2, j} w_{j}|A(t)|^{2}, \\
\frac{\partial w_{j}}{\partial t}=-\frac{w_{j}+1}{T_{j}}+\alpha_{3, j}\left(\rho_{12, j}-\rho_{12, j}^{*}\right)|A(t)|^{2} .
\end{gathered}
$$
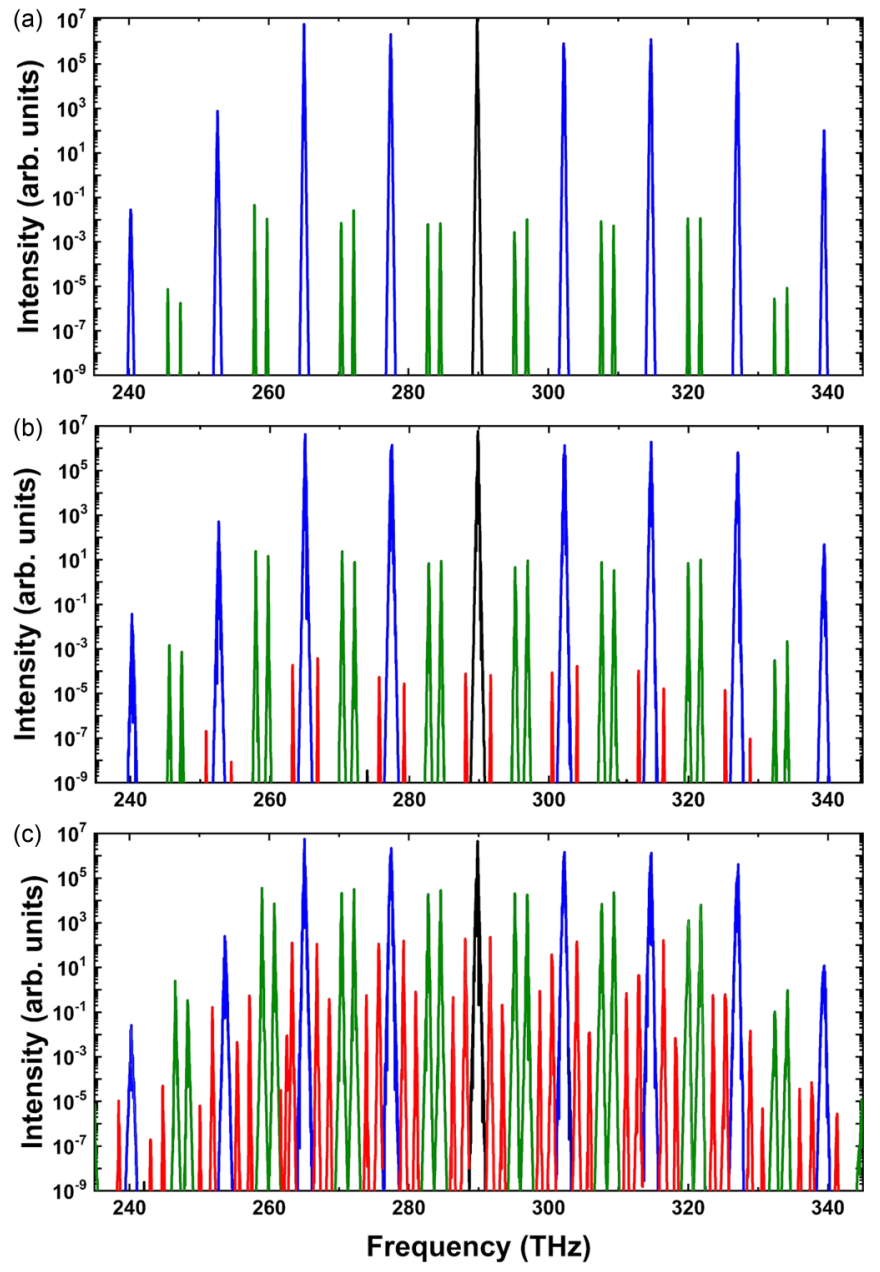

FIG. 4. (a)-(c) Evolution of the numerical output spectra for the cases of coupled pump power of $12.8 \mathrm{~W}$ (a), 14.4 W (b), and $16 \mathrm{~W}$ (c). The fiber length was $3 \mathrm{~m}$, the $\mathrm{D}_{2}$ gas pressure was 40 bars. The pump laser line is shown with black color, the ortho-ro-vibrational shifts with blue color, the para-ro-vibrational shifts with green color, and all the FWM-generated sidebands with red color.

Here $t_{j}$ and $T_{j}$ are the polarization and inversion decay times, and $\alpha_{1, j}, \alpha_{2, j}$, and $\alpha_{3, j}$ are coefficients which describe the coupling between electric field and the state of a molecule. All of the above coefficients are determined phenomenologically, as described in, e.g., [28].

A single carrier frequency along with an envelope varying on a few-fs timescale were used to treat all the comb components simultaneously and self-consistently. Figure 4 shows the calculated evolution of the output spectra for the case of coupled pump powers of $12.8 \mathrm{~W}$ [Fig. 4(a)], $14.4 \mathrm{~W}$ [Fig. 4(b)], and $16 \mathrm{~W}$ [Fig. 4(c)]. These numerical output spectra present the same pattern as the experimental one and are in a good agreement with the generation sequence of Fig. 2. The color scheme is the same as in Fig. 1, with the pump laser line $v_{0,0,0}$ shown by black color, the ortho$D_{2} v_{0, m, 0}$ lines by blue, the para- $\mathrm{D}_{2} v_{0, m, \pm 1}$ lines by green, and all the FWM sidebands $v_{F W M}$ shown by red color. Besides the very good agreement between the numerical and experimental data, the main conclusion of the numerical 
approach is the mechanism and sequence of processes, which yield the comb in a molecular Raman gas medium. In the simulation, as the propagation distance increases, an ortho$\mathrm{D}_{2}$ ro-vibrational comb (blue) appears first during Stage (i). The lines of this comb are sufficiently strong that during Stage (ii) a para- $\mathrm{D}_{2}$ ro-vibrational comb (green) appears on both sides of each line in the ortho- $\mathrm{D}_{2}$ ro-vibrational comb. Figure. 4(a) shows perfectly the generated and classic Raman comb only based on the pure Raman transitions. After that, as a third step, FWM processes set in. They include the $v_{F W M 1}=$ $v_{0, m, 0}+v_{0, m+1,-1}-v_{0, m, 1}$ type of FWM [Stage (iiia) and Fig. 4(b)] as explained above, followed by $v_{F W M 2}=v_{0, m, 1}+$ $v_{0, m, 0}-v_{F W M 1}$ and $v_{F W M 2}=v_{0, m, 1}-v_{0, m+1,-1}$ [Stage (iiib) and Fig. 4(c)]. This sequence is a cascaded one, since the $v_{F W M 1}$ is required for the $v_{F W M 2}$ to be generated. Figure 4 also confirms such a sequence, where one can see that the $v_{F W M 1}$ lines are stronger than $v_{F W M 2}$. Finally, very weak orange lines are not visible in the numerical simulations. This sequence of the elementary processes, in particular the mechanism of the generation of the red FWM lines, agrees with the analysis presented in the previous section, which shows that the small and single frequency spacing is related to the two-step Raman process, followed by cascaded parametric FWM, which is based on Kerr-type nonlinearity provided by the medium Raman response.

\section{DISCUSSION}

In this work, we have introduced a means to create a singlefrequency spaced comb, with a spacing $\Delta=v_{R 2}-2 v_{R 3}$, by driving a Raman medium in the transient regime. In view of followup works seeking to use this technique to generate a pulse-to-pulse coherent comb, we discuss the properties of intrapulse and pulse-to-pulse coherence of such a spectrum. Following the results reported in experiments [10,12,29], this comb exhibits intrapulse coherence. As a matter of fact, because, the intrapulse coherence of Raman generated spectrum is proportional to $\Gamma \tau_{p} / G_{R}$ [30], we expect higher intrapulse coherence as in $[10,12,29]$. Here, $\Gamma$ is the relaxation time of a Raman resonance and $G_{R}$ the Raman net gain (see Appendix A). Furthermore, the results in [12,29] show that the pulse-to-pulse comb is phase uncorrelated, as one would expect from an independent random process. Even though we use a much higher repetition rate and higher Raman net gain than in $[10,12]$, they are still lower than the required values to ensure that the number of molecules that are coherently excited by one pulse would remain at the required level for a coherent amplification by the following pulse [30]. This is corroborated with our numerical calculation of the shotto-shot coherence of the obtained spectrum. The latter is achieved by repeating the simulation of the input pulse shapes $A_{i}(0, \omega)$, which differ by an added random contribution [31], to mimic the condition of the Raman amplification from the spontaneously emitted Stokes field. After the propagation is simulated, the shot-to-shot coherence $g(\omega)$ is determined by the following correlation function [31]:

$$
g(\omega)=\operatorname{Re}\left[\frac{\left\langle A_{i}(z, \omega) A_{j}^{*}(z, \omega)\right\rangle_{i, j, i \neq j}}{\left\langle A_{i}(z, \omega) A_{i}^{*}(z, \omega)\right\rangle_{i}}\right] .
$$

The average coherence of the predicted spectrum was found to be 0.071 . As expected, this quite low value indicates that the $1 \mathrm{MHz}$ repetition rate of our laser is too low or that the Raman net gain is too weak. In other word, with this repetition rate and Raman net gain, each pulse amplifies his "own" Stokes wave packet from the quantum noise.

In order to ensure shot-to-shot coherence in a comb generated by the presented method, several techniques could be used. First, the use of a pump laser with stabilized carrierenvelop phase, and with sufficiently high repetition rate in order for a sufficient number of Raman excited molecules to survive the duration between two adjacent pump pulses [30], would be the closest scheme to our experiment. A second pumping scheme is similar to the one reported in [6]. It relies on exciting the medium with a pair of lasers whose frequency difference matches one of the two commensurable Raman frequencies. This removes the quantum nature of the first amplified Raman line.

In addition to the above, it is noteworthy that two pump lasers, whose frequency difference can be tuned to a value equal to a fraction of one of the two commensurable resonance frequencies, could be a means to generate an optical comb whose frequency spacing can be actively controlled by the judicious choice of the pumps frequency difference.

\section{CONCLUSION}

In conclusion, we reported on the generation of a high power, broadband, and single-frequency spaced comb based on a sequence of SRS and parametric four-wave mixing in a multiple Raman resonance molecular gas medium. The comb frequency spacing $\Delta$ results from the fact that two Raman frequencies of the gas are multiples of $\Delta$. This optical comb generation process unlocks several limitations of current comb generation techniques. It can combine the multioctave bandwidth of HC-PCF based Raman combs with single and smaller frequency spacing than in current spectra used for waveform synthesis [1] and attosecond pulse generation [8]. The results represent an important milestone towards exploring this Raman-Kerr comb into a frequency ruler [7].

\section{ACKNOWLEDGMENTS}

This work was funded by Agence Nationale de la Recherche (ANR) (PhotoSynth, Labex SigmaLim, UV factor), by Région Limousin, by the Air Force Office of Scientific Research (AFOSR) (FA9+550-14-1-0024), and by the National Science Foundation (NSF) (PHY-1068865). The authors thank the PLATINOM platform in their help in the fiber fabrication.

\section{APPENDIX A: COMPETITION BETWEEN SRS AND FWM}

In order to have a qualitative assessment of the dominant process between SRS and FWM in the generation of the red line (Fig. 1), we proceed by estimating their effective gains or alternatively their generation characteristic lengths. For both SRS and Kerr effect, we can write the following for Raman net gain:

$$
G_{R}=g_{R}\left(P_{R} / A\right) L=L L_{R}^{-1}
$$


with

$$
g_{R}\left(P_{R} / A\right)=L_{R}^{-1},
$$

and, for the Kerr process, the characteristic length required to reach the power $P_{s i}$ can be derived as [26]:

$$
L_{K}=\frac{\lambda}{2 \pi n_{2}} \sqrt{\frac{A^{2} P_{s i}}{P_{K^{3}}}} .
$$

Although the generation of a new frequency from three existing components is not a gain-type process, the following gain coefficient can be formally associated with the relevant four-wave mechanism:

$$
g_{K}=L_{K}^{-1} .
$$

Here, $P_{R}$ and $P_{K}$ are the optical powers of the exciting fields involved in SRS and FWM respectively. In the expression of the Kerr gain, we ignore the phase matching condition between the fields in the FWM. $L_{R}$ and $L_{K}$ are the generation characteristic lengths for the processes of SRS and Kerr respectively. The stronger the generation process the shorter the characteristic length. Consequently, finding out whether FWM is dominant or not is determined by the ratio $\left(L_{K} / L_{R}\right)$. The quantity $\lambda$ is the wavelength of the pumping field. $n_{2}$ is the nonlinear index of the Raman driven $\mathrm{D}_{2}$, and $g_{R}$ is the Raman gain coefficient.

Recall that in the transient regime the Raman gain coefficient $g_{R}$ is equal to $g_{R, t}$, which is related to the steady-state Raman gain coefficient $g_{R, s s}$ by [23]

$$
g_{R, t}=R \times g_{R, s s}
$$

with

$$
R=2 \sqrt{\frac{\Gamma_{\tau}}{g_{R, s s}\left(P_{R} / A\right) L}} .
$$
[24]

And, the pulse duration of the Stokes field, $\tau_{S}$ is given by

$$
\tau_{S}=\sqrt{\frac{\tau}{16 g_{R, s s} \Gamma\left(P_{R} / A\right) L}} .
$$

Here, $\Gamma$ is the Raman dephasing rate and $\tau$ is the pulse duration of the Raman pumping field.

Let us take the case of cascade SRS generation of one of the spectral lines of interest, the line at frequency $v_{0,-1,-2}$ (labeled FWM1). This is the second-order Stokes line (blue) at $v_{0,-1,0}$ or the first-order Stokes line (green) at $v_{0,-1,-1}$. Using the above expressions of the gain coefficient and the pulse duration [Eqs. (A6) and (A7)], we readily find the Raman gain coefficient for the generation of the red line $v_{0,-1,-2}$. Setting the average power of the blue line $v_{0,-1,0}$ to $1.6 \mathrm{~W}$, and that of the green line $v_{0,-1,-1}$ to $0.16 \mathrm{~W}$, which are consistent with the measured output spectrum when $16 \mathrm{~W}$ of the laser power is sent to the fiber, we find $g_{R, t} \approx 10^{-6} \mathrm{~cm} \mathrm{GW}^{-1}$ and $L_{R} \approx 495 \mathrm{~cm}$ for the generation of the red line $\nu_{0,-1,-2}$.

Similarly, we estimate the Kerr gain and characteristic length by setting the pumping average power to $1.6 \mathrm{~W}$, and taking $n_{2}=3 \times 10^{-8} \mathrm{~cm}^{2} / \mathrm{TW}$, the value estimated as explained in the text of the paper [27]. We found $g_{K} \approx 3.8 \times$ $10^{-6} \mathrm{~cm} \mathrm{GW}^{-1}$ and $L_{K} \approx 4.5 \mathrm{~cm}$.
These approximate calculations show that $L_{R}$ is 110 times larger than $L_{K}$, thus indicating that Kerr dominates the red line $v_{0,-1,-2}$. A similar approach shows that this is valid for all red lines.

\section{APPENDIX B: SUSCEPTIBILITY IN RAMAN-ACTIVE MEDIUM}

The full expression for the susceptibility in the Ramanactive medium is discussed. Our derivation of the expression for the nonlinear susceptibility of the Raman medium follows the general line and denotations of Ref. [28]. We begin the derivation of this expression by considering a system which can be described by a three-level system with two lower levels $|a\rangle$ and $|b\rangle$ with energies $\hbar \omega_{a}$ and $\hbar \omega_{b}, \omega_{a}<\omega_{b}$, and higher level $|j\rangle$ with energy $\hbar \omega_{j}$. Without the loss of generality we set $\omega_{a}=0$ and $\omega_{a}=\omega_{R}$, where $\omega_{R}$ is the Raman shift. The higher level $j$ can be understood as a combination of multiple higher levels. The external field has three frequency components:

$$
E(t)=\frac{1}{2} \sum_{j=1,3} E_{j} e^{-i \omega_{j} t}+\text { c.c. },
$$

which couple the energy levels of the system through the Hamiltonian

$$
H=\left(\begin{array}{ccc}
0 & 0 & V_{a j} \\
0 & \hbar_{\omega_{R}} & V_{b j} \\
V_{j a} & V_{j b} & \hbar_{\omega_{j}}
\end{array}\right)
$$

Here

$$
\begin{aligned}
& V_{a j}=V_{j a}^{*}=-\mu_{a j} E, \\
& V_{b j}=V_{j b}^{*}=-\mu_{b j} E .
\end{aligned}
$$

As one can see from these expressions, the levels $|a\rangle$ and $|j\rangle$ as well as $|b\rangle$ and $|j\rangle$, are coupled by electric dipole transitions, while the transition between $|a\rangle$ and $|b\rangle$ is forbidden.

In this case, the amplitudes $C_{n}$ of the wave function of the system

$$
|\psi\rangle=\sum_{n=a, b, j} C_{n} e^{-i \omega_{n} t}|n\rangle
$$

satisfy the following equations which follow from the Schrödinger equation:

$$
\frac{\partial C_{n}}{\partial t}=-\frac{i}{\hbar} \sum_{k} e^{-\left(\omega_{n}-\omega_{k}\right) t} V_{n k} C_{k}
$$

This set of linear equations is solved by the usual methods, utilizing the adiabatic elimination of $C_{j}$ [28]. The details of the derivation are standard and too cumbersome to be presented here. After the expressions for the coefficients $C_{n}$ are obtained, the polarization of the medium can be expressed through

$$
P=N \sum_{n=a, b} \mu_{j a} C_{a} C_{j}^{*} e^{-i\left(\omega_{a}-\omega_{j}\right)}+\text { c.c., }
$$

$N$ being the particle density.

The final expression for the polarization reads

$$
P(t)=\frac{N \mu_{j a}^{2}}{64 \hbar^{3}}\left(\left|\mu_{j a}\right|^{2} A+\left|\mu_{j b}\right|^{2} B\right),
$$


where

$$
\begin{aligned}
A= & \left\{\sum_{q, q^{\prime}}\left[-\frac{E_{q} E_{q^{\prime}} e^{-i\left(\omega_{q}-\omega_{q^{\prime}}\right) t}}{\left(\omega_{q}+\omega_{q^{\prime}}\right)\left(\omega_{j}-\omega_{q^{\prime}}\right)}+\frac{E_{q}^{*} E_{q^{\prime}}^{*} e^{-i\left(\omega_{q}-\omega_{q^{\prime}}\right) t}}{\left(\omega_{q}-\omega_{q^{\prime}}\right)\left(\omega_{j}-\omega_{q^{\prime}}\right)}\right]\right. \\
& \left.+\sum_{q, q^{\prime} \neq q}\left[-\frac{E_{q} E_{q^{\prime}}^{*} e^{-i\left(\omega_{q}-\omega_{q^{\prime}}\right) t}}{\left(\omega_{q}-\omega_{q^{\prime}}\right)\left(\omega_{j}+\omega_{q^{\prime}}\right)}+\frac{E_{q}^{*} E_{q^{\prime}} e^{-i\left(\omega_{q}-\omega_{q^{\prime}}\right) t}}{\left(\omega_{q}-\omega_{q^{\prime}}\right)\left(\omega_{j}-\omega_{q^{\prime}}\right)}\right]+\text { c.c. }\right\}\left\{\sum_{q^{\prime \prime}}\left[\frac{E_{q^{\prime \prime}} e^{-i \omega_{q^{\prime \prime}} t}}{\omega_{j}-\omega_{q^{\prime \prime}}}+\frac{E_{q^{\prime \prime}}^{*}}{\omega_{j}+\omega_{q^{\prime \prime}}}\right]+\text { c.c. }\right\}
\end{aligned}
$$

and

$$
\begin{aligned}
B= & \left\{\sum_{q, q^{\prime}}\left[-\frac{E_{q} E_{q^{\prime}} e^{-i\left(\omega_{q}+\omega_{q^{\prime}}\right) t}}{\left(\omega_{R}-\omega_{q}-\omega_{q^{\prime}}\right)\left(\omega_{j}-\omega_{q^{\prime}}\right)}+\frac{E_{q}^{*} E_{q^{\prime}}^{*} e^{i\left(\omega_{q}+\omega_{q^{\prime}}\right) t}}{\left(\omega_{R}+\omega_{q}+\omega_{q^{\prime}}\right)\left(\omega_{j}+\omega_{q^{\prime}}\right)}\right]\right. \\
& \left.+\sum_{q, q^{\prime} \neq q}\left[-\frac{E_{q} E_{q^{\prime}}^{*} e^{-i\left(\omega_{q}-\omega_{q^{\prime}}\right) t}}{\left(\omega_{R}-\omega_{q}+\omega_{q^{\prime}}\right)\left(\omega_{j}+\omega_{q^{\prime}}\right)}+\frac{E_{q}^{*} E_{q^{\prime}} e^{i\left(\omega_{q}-\omega_{q^{\prime}}\right) t}}{\left(\omega_{R}+\omega_{q}-\omega_{q^{\prime}}\right)\left(\omega_{j}-\omega_{q^{\prime}}\right)}\right]+\text { c.c. }\right\} \\
& \times\left\{\sum_{q^{\prime \prime}}\left[\frac{E_{q^{\prime \prime}} e^{-i \omega_{q^{\prime \prime}} t}+\frac{E_{q^{\prime \prime}}^{*}}{\omega_{j}-\omega_{R}-\omega_{q^{\prime \prime}}}+\frac{E_{q^{\prime \prime}}^{*}}{\omega_{j}-\omega_{R}+\omega_{q^{\prime \prime}}}+\frac{E_{q^{\prime \prime}}}{\omega_{j}-\omega_{q^{\prime \prime}}}+i \omega_{q^{\prime \prime}}}{\omega_{j}+\omega_{q^{\prime \prime}}}\right]+\text { c.c. }\right\}
\end{aligned}
$$

In this expression, indices $q, q^{\prime}, q^{\prime \prime}$ denote pump fields and can take values from 1 to 3 . In deriving this expression, we have neglected the decay rates which are for our case much lower than the few-THz difference between frequencies. This expression includes all contributions provided by the Raman nonlinearity, including Raman contributions to selfand cross-phase modulation and in particular four-wave mixing. Equation (B10) contains in total 1440 terms, therefore in the main text we present a susceptibility corresponding to only one of these contributions. Since the values of dipole momenta $\mu_{j a}$ and $\mu_{j a}$ are similar, the dominant terms are determined by the smallest value of the denominator. For typical conditions, $\omega_{j}=\omega_{R}$ and $\omega_{j}=\omega_{q}$, therefore the factors in the denominator which contain $\omega_{j}$ will have similar values for all terms, with terms containing $\omega_{j}-\omega_{q}$ in the denominator being larger. On the other hand, the terms in $A$ which contain in the denominator the combination $\omega_{q}-\omega_{q^{\prime}}$, and the terms in $B$ which contain in the denominator combinations $\omega_{R} \pm\left(\omega_{q}-\omega_{q^{\prime}}\right)$, are larger than other terms and will provide dominant contributions to the respective nonlinear processes. Considering the above arguments, we identify the dominant contributions $A_{\mathrm{dom}}$ and $B_{\mathrm{dom}}$ to $A$ and $B$ as follows:

$$
\begin{aligned}
A_{\mathrm{dom}}= & \left\{\sum_{q, q^{\prime} \neq q} \frac{E_{q}^{*} E_{q^{\prime}} e^{i\left(\omega_{q}-\omega_{q^{\prime}}\right) t}}{\left(\omega_{q}-\omega_{q^{\prime}}\right)\left(\omega_{j}-\omega_{q^{\prime}}\right)}+\text { c.c. }\right\} \\
& \times\left\{\sum_{q^{\prime \prime}} \frac{E_{q^{\prime \prime}} e^{-i \omega_{q^{\prime \prime}} t}}{\omega_{j}-\omega_{q^{\prime \prime}}}+\text { c.c. }\right\}
\end{aligned}
$$

and

$$
\begin{aligned}
B_{\mathrm{dom}}= & \left\{\sum_{q, q^{\prime}} \frac{E_{q} E_{q^{\prime}} e^{i\left(\omega_{q}-\omega_{q^{\prime}}\right) t}}{\left(\omega_{R}-\omega_{q}-\omega_{q^{\prime}}\right)\left(\omega_{j}-\omega_{q^{\prime}}\right)}\right. \\
& \left.+\sum_{q, q^{\prime}} \frac{E_{q}^{*} E_{q^{\prime}} e^{i\left(\omega_{q}-\omega_{q^{\prime}}\right) t}}{\left(\omega_{R}+\omega_{q}-\omega_{q^{\prime}}\right)\left(\omega_{j}-\omega_{q^{\prime}}\right)}+\text { c.c. }\right\} \\
& \times\left\{\sum_{q^{\prime \prime}}\left[\frac{E_{q^{\prime \prime}} e^{-i \omega_{q^{\prime \prime}} t}}{\omega_{j}-\omega_{R}-\omega_{q^{\prime \prime}}}+\frac{E_{q^{\prime \prime}}^{*} e^{i \omega_{q^{\prime \prime}} t}}{\omega_{j}-\omega_{q^{\prime \prime}}}\right]+\text { c.c. }\right\}
\end{aligned}
$$

[1] H.-S. Chan, Z.-M. Hsieh, W.-H. Liang, A. H. Kung, C.-K. Lee, C.-J. Lai, R.-P. Pan, and L.-H. Peng, Synthesis and measurement of ultrafast waveforms from five discrete optical harmonics, Sci. 331, 1165 (2011).

[2] S. B. Papp, K. Beha, P. Del'Haye, F. Quinlan, H. Lee, K. J. Vahala, and S. A. Diddams, Microresonator frequency comb optical clock, Optica 1, 10 (2014).

[3] J. Pfeifle, V. Brasch, M. Lauermann, Y. Yu, D. Wegner, T. Herr, K. Hartinger, P. Schindler, J. Li, D. Hillerkuss, R. Schmogrow, C. Weimann, R. Holzwarth, W. Freude, J. Leuthold, T. J. Kippenberg, and C. Koos, Coherent terabit communications with microresonator Kerr frequency combs, Nat. Photonics 8, 375 (2014).
[4] T. Ideguchi, S. Holzner, B. Bernhardt, G. Guelachvili, N. Picque, and T. W. Hansch, Coherent Raman spectro-imaging with laser frequency combs, Nature (London) 502, 355 (2013).

[5] H. W. Mocker and R. J. Collins, Mode competition and selflocking effects in a Q-switched ruby laser, Appl. Phys. Lett. 7, 270 (1965).

[6] S. E. Harris and A. V. Sokolov, Broadband spectral generation with refractive index control, Phys. Rev. A 55, R4019 (1997).

[7] T. Udem, R. Holzwarth, and T. W. Hänsch, Optical frequency metrology, Nature (London) 416, 233 (2002).

[8] F. Krausz and M. Ivanov, Attosecond physics, Rev. Mod. Phys. 81, 163 (2009). 
[9] P. Del'Haye, A. Schliesser, O. Arcizet, T. Wilken, R. Holzwarth, and T. J. Kippenberg, Optical frequency comb generation from a monolithic microresonator, Nature (London) 450, 1214 (2007).

[10] F. Couny, F. Benabid, P. J. Roberts, P. S. Light, and M. G. Raymer, Generation and photonic guidance of multi-octave optical-frequency combs, Science 318, 1118 (2007).

[11] A. Benoît, B. Beaudou, M. Alharbi, B. Debord, F. Gerome, F. Salin, and F. Benabid, Over-five octaves wide Raman combs in high-power picosecond-laser pumped $\mathrm{H}_{2}$-filled inhibited coupling Kagome fiber, Opt. Exp. 23, 14002 (2015).

[12] Y. Y. Wang, C. Wu, F. Couny, M. G. Raymer, and F. Benabid, Quantum-Fluctuation-Initiated Coherence in Multioctave Raman Optical Frequency Combs, Phys. Rev. Lett. 105, 123603 (2010).

[13] P. Hosseini, A. Abdolvand, and P. St. J. Russell, Generation of spectral clusters in a mixture of noble and Raman-active gases, Opt. Lett. 41, 5543 (2016).

[14] F. Tani, F. Belli, A. Abdolvand, J. C. Travers, and P. S. Russell, Generation of three-octave-spanning transient Raman comb in hydrogen-filled hollow-core PCF, Opt. Lett. 40, 1026 (2015).

[15] Y. R. Shen and N. Bloembergen, Theory of Stimulated Brillouin and Raman scattering, Phys. Rev. 137, A1787 (1965).

[16] R. W. Boyd, M. G. Raymer, P. Narum, and D. J. Harter, Four-wave parametric interactions in a strongly driven two-level systems, Phys. Rev. A. 24, 411 (1981).

[17] F. Benabid, J. C. Knight, G. Antonopoulos, and P. S. J. Russell, Stimulated Raman scattering in hydrogen-filled hollow-core photonic crystal fiber, Science 298, 399 (2002).

[18] A. Benoît, E. Ilinova, B. Beaudou, B. Debord, F. Gérôme, and F. Benabid, Spectral-temporal dynamics of high power Raman picosecond pulse using $\mathrm{H}_{2}$-filled Kagome HC-PCF, Opt. Lett. 42, 3896 (2017).

[19] R. Z. Martínez, D. Bermejo, P. Wcisło, and F. Thibault, Accurate wavenumber measurements for the $S_{0}(0), S_{0}(1)$, and $S_{0}(2)$ pure rotational Raman lines of $\mathrm{D}_{2}$, J. Raman Spectrosc. 50, 127 (2019).
[20] D. K. Veirs and G. M. Rosenblatt, Raman line positions in molecular hydrogen: $\mathrm{H}_{2}, \mathrm{HD}, \mathrm{HT}, \mathrm{D}_{2}, \mathrm{DT}$, and $\mathrm{T}_{2}$, J. Mol. Spectrosc. 121, 401 (1987).

[21] A. Maczek and A. Meijer, Statistical Thermodynamics, Oxford Chemistry Primers Series (Oxford University Press, Oxford, 2017), pp. 63-64.

[22] B. Debord, A. Amsanpally, M. Chafer, A. Baz, M. Maurel, J.-M. Blondy, E. Hugonnot, F. Scol, L. Vincetti, F. Gerome, and F. Benabid, Ultralow transmission loss in inhibited-coupling guiding hollow fibers, Optica 4, 209 (2017).

[23] R. J. Heeman and H. P. Godfried, Gain reduction measurements in transient stimulated Raman scattering, IEEE J. Quantum Electron. 31, 358 (1995).

[24] M. G. Raymer, I. A. Walmsley, J. Mostowski, and B. Sobolewska, Quantum theory of spatial and temporal coherence properties of stimulated Raman scattering, Phys. Rev. A 32, 332 (1985).

[25] Y. Okawachi, M. Yu, V. Venkataraman, P. M. Latawiec, A. G. Griffith, M. Lipson, M. Lončar, and A. L. Gaeta, Competition between Raman and Kerr effects in microresonator comb generation, Opt. Lett. 42, 2786 (2017).

[26] R. W. Boyd, Nonlinear Optics, 3rd ed. (Academic, New York, 2003).

[27] S. Xu, B. Lavorel, O. Faucher, and R. Chaux, Characterization of self-phase modulated ultrashort optical pulses by spectral phase interferometry, J. Opt. Soc. Am. B 19, 165 (2002).

[28] V. P. Kalosha and J. Herrmann, Phase Relations, Quasicontinuous Spectra and Subfemtosecond Pulses in High-Order Stimulated Raman Scattering with Short-Pulse Excitation, Phys. Rev. Lett. 85, 1226 (2000).

[29] C. Wu, M. G. Raymer, Y. Y. Wang, and F. Benabid, Quantum theory of phase correlations in optical frequency combs generated by stimulated Raman scattering, Phys. Rev. A. 82053834 (2010).

[30] D. T. Smithey, M. Belsley, K. Wedding, and M. G. Raymer, Near Quantum-Limited Phase Memory in a Raman Amplifier, Phys. Rev. Lett. 67, 2446 (1991).

[31] J. M. Dudley, G. Genty, and S. Coen, Supercontinuum generation in photonic crystal fiber, Rev. Mod. Phys. 78, 1135 (2006). 